\title{
LOW PRESSURE DROP MICRO PRECONCENTRATORS WITH A COBWEB TENAX-TA FILM

\author{
B. Alfeeli ${ }^{1,2^{*}}$ and M. Agah ${ }^{1}$
} \\ ${ }^{1}$ Virginia Polytechnic Institute and State University, Blacksburg, Virginia, USA \\ ${ }^{2}$ Kuwait Institute for Scientific Research, KUWAIT
}

\begin{abstract}
This paper reports the design, simulation, and experimental evaluation of high performance low pressure drop $(<100 \mathrm{~Pa} / \mathrm{cm})$ micropreconcentrator $(\mu \mathrm{PC})$ chips $(7 \mathrm{~mm} \times 7 \mathrm{~mm} \times 0.38 \mathrm{~mm})$ for micro total analytical systems. The $\mu$ PC's high performance is attributed to the multiple inlets/outlets, novel high-aspect-ratio $(380 \mu \mathrm{m})$ parabolic reflectors, and cobweb configuration of adsorbent films all in which promote the adsorption of chemical species. Flow pattern simulations were used to verify the underlying concept of the design. The adsorption capacity of hydrocarbons was used to evaluate the $\mu \mathrm{PCs}$ experimentally. The performance of the new design was found to be more than 2 orders of magnitude higher than the first generation reported previously.
\end{abstract}

\section{INTRODUCTION}

Generally speaking, preconcentrators (PCs) are concentration amplifiers used to improve the detection limit of analytical systems such as spectroscopy [1], mass-spectrometry [2], and chromatography [3]. They are also an integral part of handheld chemical detection systems. However, to meet the size and power constraints, handheld systems rely on micofabricated components (fluidic ports/channels, pumps, valves, etc.) which operate at low pressures. For example, a state-of-the-art micromachined pump designed for micro gas chromatographers can only provide a maximum back pressure of $7 \mathrm{kPa}$ [4] whereas a typical MEMSbased $\mu \mathrm{PC}$ can introduce large pressure drop as high as $14 \mathrm{kPa}$ [5]. Therefore, reducing the pressure drop in $\mu \mathrm{PCs}$ is a key development in handheld systems.

Conventional PCs are troubled by large pressure drop, dead volume, and high power consumption. Microfabrication technology has enabled high efficiency preconcentration in terms of sample capacity, device size, and the energy consumed. Recently, our group and others have utilized 3D microstructures embedded within a microcavity and coated with adsorbent films to realize low pressure drop $\mu \mathrm{PC}$ [6-8]. The microstructures provide large surface-to-volume ratio and low flow resistance compared to conventional microporous granular packing arrangement. This paper reports the design, simulation, and experimental evaluation of high performance low pressure drop $\mu \mathrm{PC}$ chips for micro analytical instruments including a micro gas chromatography system $(\mu \mathrm{GC})$ [9]. In addition to our unique design enabling very low pressure drops, less than $100 \mathrm{~Pa} / \mathrm{cm}$, we present a new deposition method which results in a cobweb structure of the adsorbent material, thereby significantly increasing the concentration efficiency without obstructing the gas flow.

The new design consists of an array of parabolic reflectors embedded within a $7 \mathrm{~mm} \times 7 \mathrm{~mm} \times 0.38 \mathrm{~mm}$ cavity. The unit cell of the array consists of three reflectors as shown in Fig. 1. The reflectors are arranged to split the flow in the forward direction and combine them back in the reverse direction. The spacing between the reflectors (side and middle spacing) was varied to investigate its effect on the device performance. Table 1 list the different spacing values for both side and middle spacing along with the symmetry ratios (SR) of different designs. The SR was defined as the ratio of side to middle spacing. High SR represents narrow passage of flow which increases the probability of molecules scattering and interacting with the adsorption surface. Full scale (actual chip size) computational fluid dynamics (CFD) simulation was consummated to investigate the flow behavior in such geometries. To establish confidence in the CFD model, calculated flow rates were compared with experimental results as shown in Fig. 2. The CFD results of the flow velocity profile showed high velocity in the middle spacing region of $\mathrm{PC} 100$ design as presented in Fig. 3. The data suggested that high SR value is desirable. The CFD results confirmed the anticipated flow behaviors. The simulated pressure drops in the different designs are listed in Table 1. The pressure drop profile is shown in Fig. 4 indicates uniform pressure drop which is also desirable.

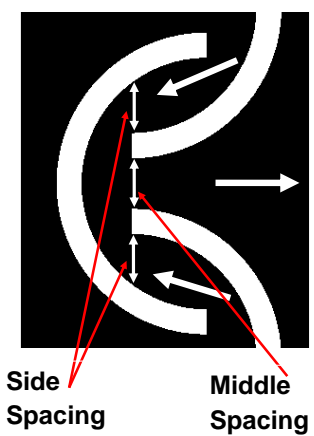

Figure 1: The $\mu$ PC unit cell. Arrows indicate the flow direction.

Table 1: Different spacing arrangements and their corresponding symmetry ratio along with the simulated pressure drops at $1.5 \mathrm{~mL} / \mathrm{min}$ flow rate.

\begin{tabular}{lcccc}
\hline $\begin{array}{l}\text { Design } \\
\text { Label }\end{array}$ & $\begin{array}{l}\text { Middle } \\
\text { spacing } \\
(\boldsymbol{\mu m})\end{array}$ & $\begin{array}{l}\text { Side } \\
\text { Spacing } \\
(\boldsymbol{\mu m})\end{array}$ & SR & $\begin{array}{l}\text { Pressure } \\
\text { drop (Pa) }\end{array}$ \\
\hline PC100 & 100 & 250 & 2.5 & 145 \\
PC200 & 200 & 200 & 1 & 97 \\
PC300 & 300 & 150 & 0.5 & 94 \\
\hline
\end{tabular}

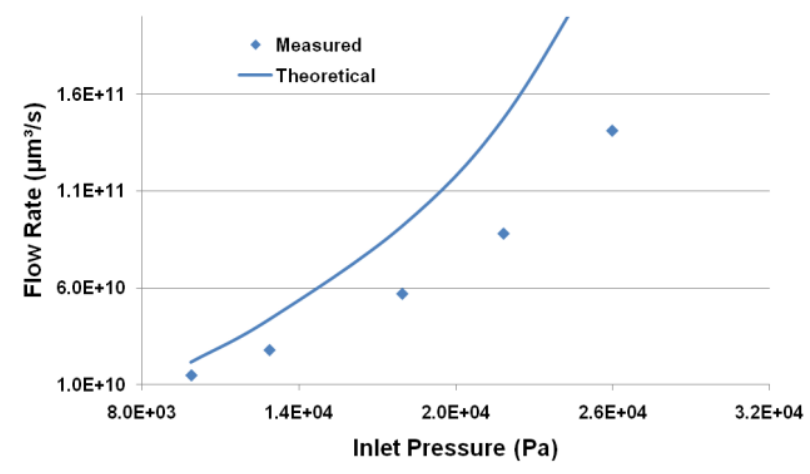

Figure 2: Comparison of calculated and experimental flow rates to establish confidence in the CFD model 


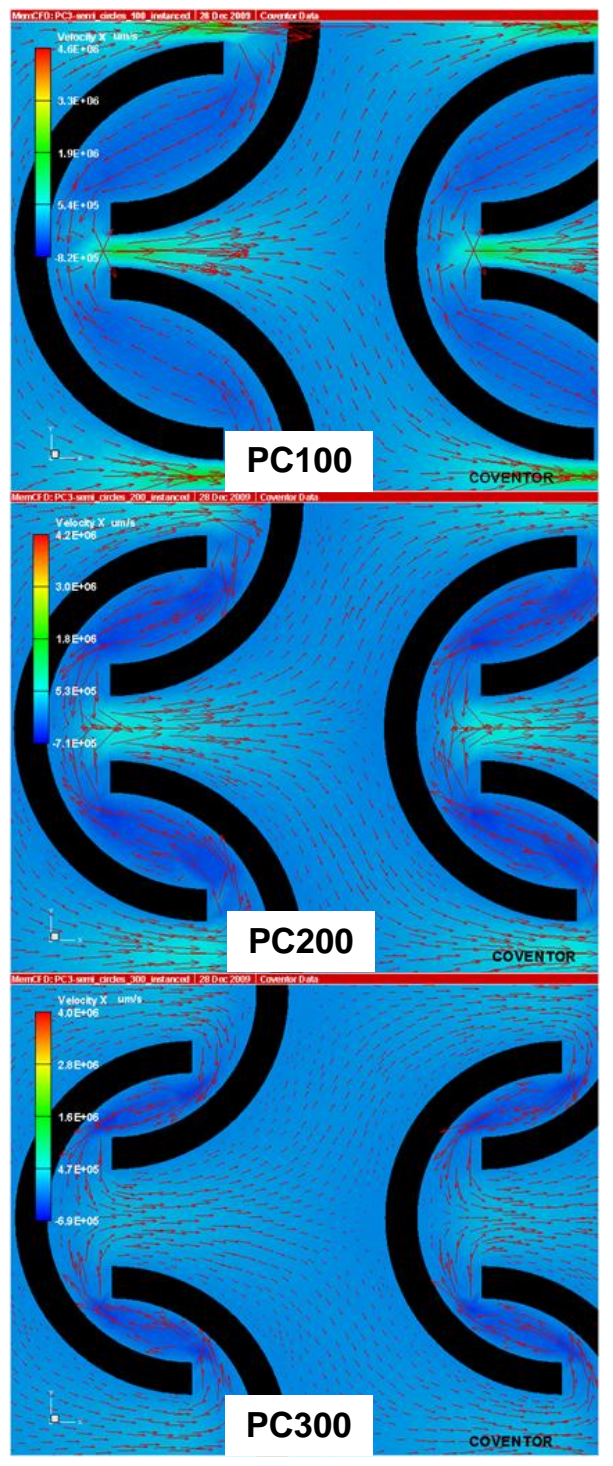

Figure 3: Simulation of the flow profile through different design spacing arrangements

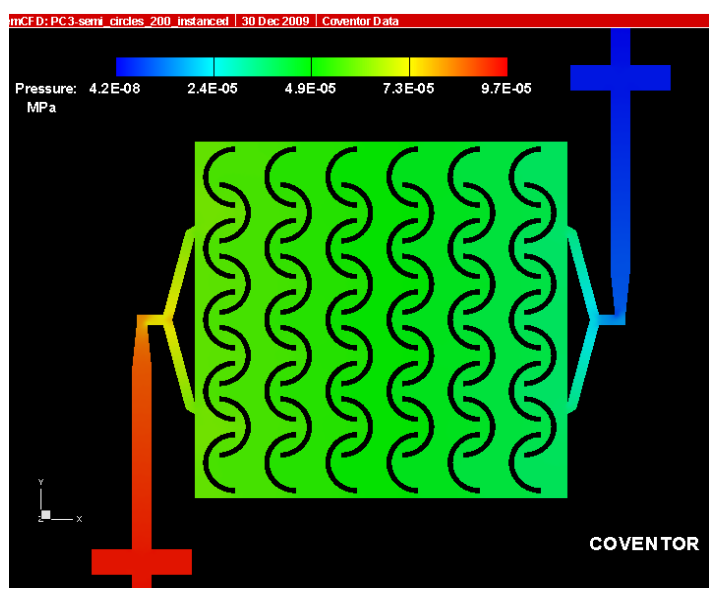

Figure 4: Pressure-drop profile in the $\mu P C$
The microstructures were realized according to the process flow in Fig. 5. The process starts with (a) photolithography of microstructures and fluidic ports on 4 inches test grade silicon wafer using high resolution, superior aspect ratio thick film photoresist (PR9260). This step was followed by (b) etching the patterned wafer using deep reactive ion etching (DRIE) to form $380 \mu \mathrm{m}$ deep 3D structures. Tenax-TA particles were dissolved in dichloromethane at $50 \mathrm{mg} / \mathrm{mL}$ concentration to create a solution to fill the etched cavity with. Adsorbent deposition (c) was achieved by adding drops of 2-propanol into solution before it evaporates from the cavity. Cobweb Tenax-TA evolves when 2-propanol contacts Tenax-TA solution. The cobweb structure anchors to the sidewalls once the solvent evaporates as shown in Fig. 6. The cavity was then sealed (d) by anodic bonding the silicon substrate to a Pyrex wafer at $1250 \mathrm{~V}$ and $300^{\circ} \mathrm{C}$ instead of conventional $350-$ $400^{\circ} \mathrm{C}$ bonding temperature to avoid damaging the polymer. The expansion reservoirs shown in Fig. 7 were incorporated into the inlet/outlet design to prevent the sealing adhesive from overflowing into the inner cavity. This was a new addition to the first generation design reported previously [7]. The silicon-Pyrex stack was then diced into individual devices like the one shown in Fig. 7. Each device was connected on both sides with $300 \mu \mathrm{m}-\mathrm{OD}$, $170 \mu \mathrm{m}$-ID deactivated fused silica tubing using high temperature ceramic based adhesive. The addition of Tenax-TA did not create a measurable difference in the flow rate. Fig. 8 presents a scanning electron microscopy (SEM) image of cobweb Tenax-TA
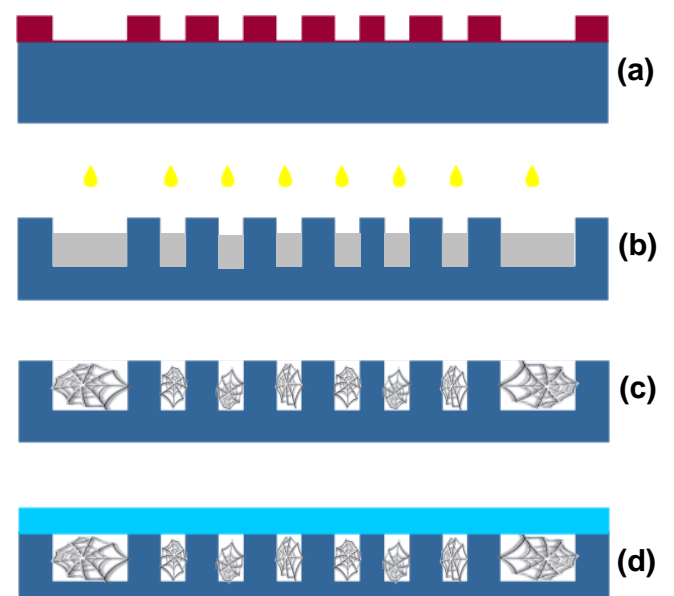

Figure 5: $\mu$ PC fabrication process flow

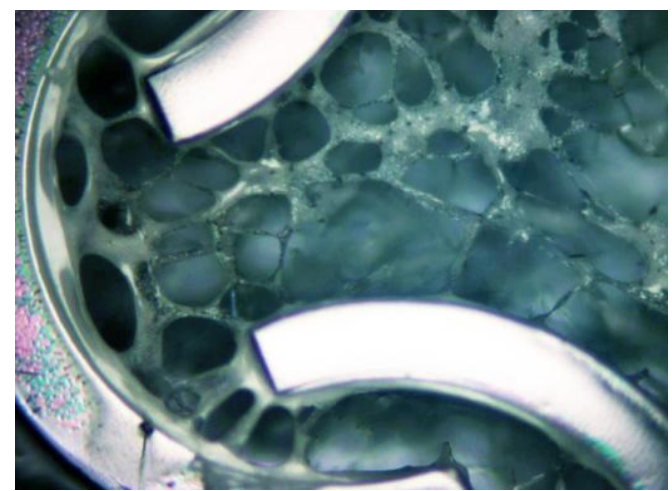

Figure 6: Optical image of the cobweb Tenax-TA. 




Figure 7: $3 D$ rendering of the $\mu P C$, insets are SEM micrographs of the fabricated microstructures

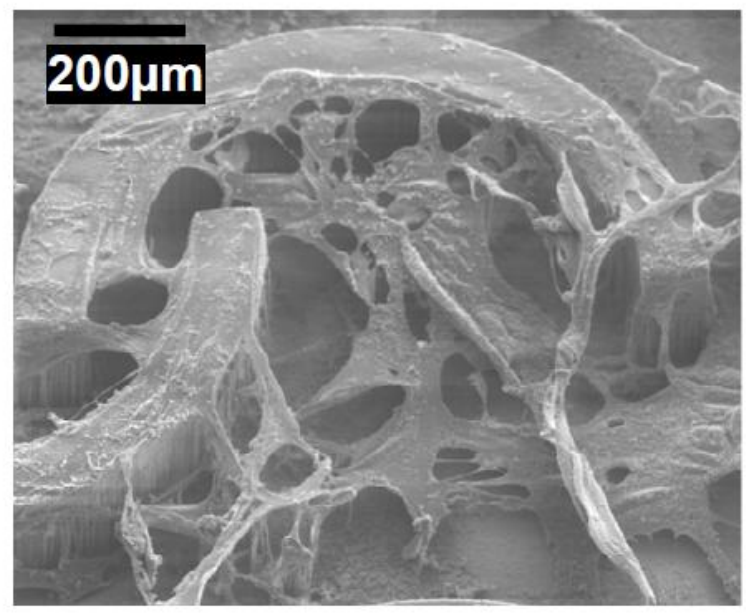

Figure 8: SEM image of cobweb Tenax-TA on the micro parabolic reflectors

The $\mu$ PCs were evaluated experimentally by using the adsorption capacity as a figure of merit. The testing was performed inside a temperature controlled chamber to accurately maintain the testing temperature at $35^{\circ} \mathrm{C}$. An electronic gas flow controller was used to supply and control the carrier gas which was selected to be nitrogen to resemble actual field operation conditions. The flow rate through the $\mu \mathrm{PC}$ was set to constant 1 $\mathrm{mL} / \mathrm{min}$. A flame ionization detector (FID) was utilized to detect the hydrocarbon samples used in the experiments. An off-board high-performance ceramic heater (ultra-fast ramp rate) was used for thermal desorption along with K-type thermocouple for manual temperature monitoring and control. In future work, on-chip thermal desorption capability will be added to the $\mu$ PCs. The chips were saturated with benzene at $35^{\circ} \mathrm{C}$ and then desorbed at $100^{\circ} \mathrm{C} / \mathrm{sec}$ to $250^{\circ} \mathrm{C}$. The FID peak area is proportional to the analyte concentration. The benzene concentration in the desorption peak reflects the sample amount collected by the device. It is evident from Fig. 9 that the SR parameter has a significant effect on the performance of the parabolic reflectors design. Moreover, Fig. 9 shows that the performance of PC100 design is more than 2 orders of magnitude higher than the micro pillar design presented in HiltonHead'08 [10]. In the first generation design inkjet printing of Tenax-TA was used to provide uniform film deposition. Cobweb Tenax-TA offers better utilization of the space between the microstructures with the $\mu \mathrm{PC}$ cavity. Reproducibility of the results in terms of peak area was $10 \%$ relative standard deviation (RSD) with more than 10 manual injections is shown in Fig. 10.

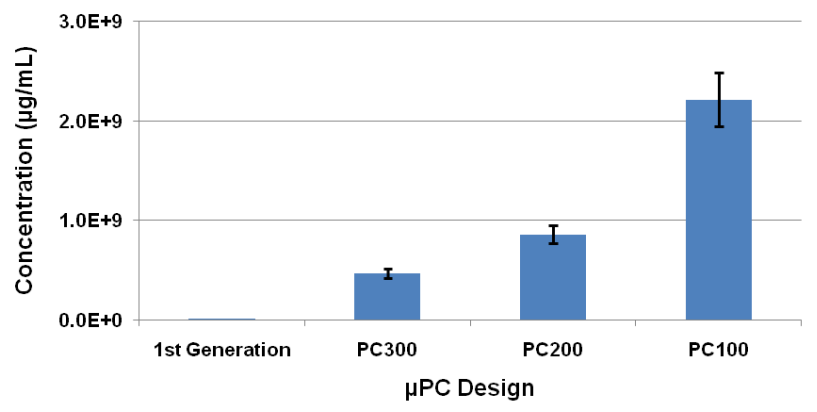

Figure 9: Benzene desorption concentration for the three designs as well as the first generation design

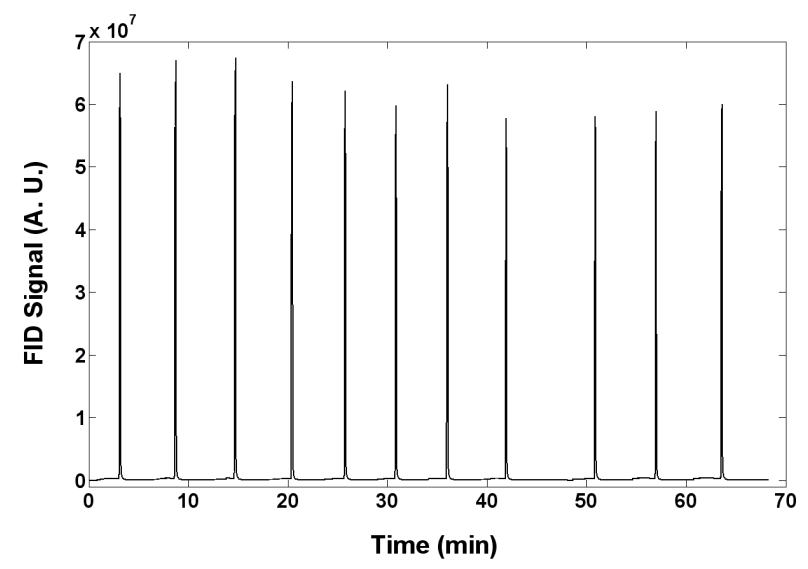

Figure 10: Reproducibility of $\mu P C$ desorption pulses

For proof of concept, the $\mu \mathrm{PC}$ was put in series with a $1 \mathrm{~m}$ $100 \%$ methylpolysiloxane microfabricated column [11] to demonstrate the possibility of $\mu \mathrm{PC}-\mu \mathrm{GC}$ integration. Under the same loading/desorbing conditions as the benzene test, the $\mu \mathrm{PC}$ was loaded with a mixture of toluene, nonane, decane, undecane, dodecane, tridecane, tetradecane, and pentadecane diluted in pentane at $1 \mathrm{ppm}$ concentrations. The sample concentration was below the FID detection limit; thus, preconcentraion was needed to make the sample detectable. Fig. 11 illustrates a chromatogram of the preconcentrated sample injected into the micro column via thermal desorption. The initial and final temperatures of the column were $35{ }^{\circ} \mathrm{C}$ and $150{ }^{\circ} \mathrm{C}$ at $30^{\circ} \mathrm{C} / \mathrm{min}$. The carrier flow rate was $1 \mathrm{~mL} / \mathrm{min}$.

\section{CONCLUSIONS}

The performance of analytical instruments especially handheld systems can be improved with sample pretreatment techniques such as preconcentration. This paper has demonstrated the possibility of developing lower pressure drops, small sizes, and high performance MEMS-based $\mu$ PCs. This was realized by utilizing findings from previous designs, novel parabolic reflectors configuration, and cobweb Tenax-TA. The simulation analyses verified by experimental results have shown the effect of the SR parameter in the parabolic reflectors design. Future work will aim to better understand and model the behavior of the $\mu$ PCs under different conditions. The reproducible desorption peaks needed for fast on-field analytical analysis has been demonstrated by the $\mu \mathrm{PC}$. 
The presented device was successful in concentrating a diluted multi-compound hydrocarbon mixture.

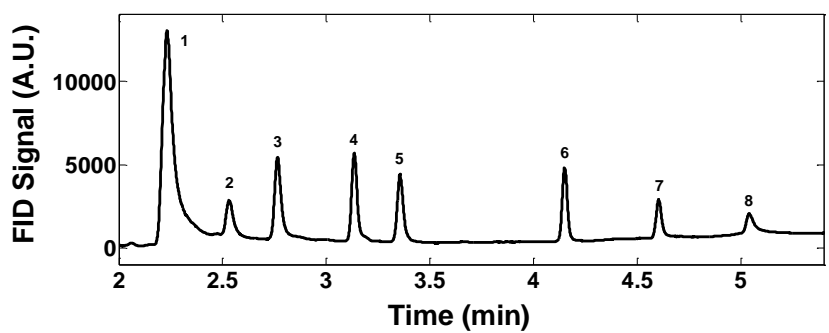

Figure 11: Preconcentration and separation of a mixture of (1) toluene, (2) nonane, (3) decane, (4) undecane, (5) dodecane, (6) tridecane, (7) tetradecane, and (8) pentadecane diluted in pentane below the FID detection limit.

\section{ACKNOWLEDGMENT}

The authors thank Mr. Richard Wilson for his assistance in creating the mask layout. This research has been primarily supported by the National Science Foundation under Award No CBET-0854242. The authors also express their appreciation to Virginia Tech Center for Microelectronics, Optoelectronics, and Nanotechnology (MicrON) and Nanoscale Characterization and Fabrication Laboratory (NCFL) of Virginia Tech Institute for Critical Technology and Applied Science (ICTAS) for assisting with the fabrication and characterization presented in this work.

\section{REFERENCES}

[1] W. A. Groves, E. T. Zellers, and G. C. Frye, "Analyzing organic vapors in exhaled breath using a surface acoustic wave sensor array with preconcentration: Selection and characterization of the preconcentrator adsorbent," Analytica Chimica Acta, 371 (2-3) pp. 131-143, 1998.

[2] C. Hornuss, S. Praun, J. Villinger, A. Dornauer, P. Moehnle, M. Dolch, E. Weninger, A. Chouker, C. Feil, J. Briegel, M. Thiel, and G. Schelling, "Real-time Monitoring of Propofol in Expired Air in Humans Undergoing Total Intravenous Anesthesia," Anesthesiology, 106 (4) pp. 665-674, 2007.

[3] K. Dettmer and W. Engewald, "Adsorbent materials commonly used in air analysis for adsorptive enrichment and thermal desorption of volatile organic compounds," Analytical and Bioanalytical Chemistry, 373 (6) pp. 490-500, 2002.

[4] A. A. Astle, H. S. Kim, L. P. Bernal, K. Najafi, and P. D. Washabaugh, "Theoretical and experimental performance of a high frequency gas micropump," Sensors and Actuators A: Physical, 134 (1) pp. 245-256, 2007.

[5] W.-C. Tian, S. W. Pang, L. Chia-Jung, and E. T. Zellers, "Microfabricated preconcentrator-focuser for a microscale gas chromatograph," Microelectromechanical Systems, Journal of, 12 (3) pp. 264-272, 2003.

[6] J. Yeom, D. D. Agonafer, J.-H. Han, and M. A. Shannon, "Low Reynolds number flow across an array of cylindrical microposts in a microchannel and figure-of-merit analysis of micropost-filled microreactors," J. Micromech. Microeng., 19 (6) p. 065025, 2009.

[7] B. Alfeeli, D. Cho, M. Ashraf-Khorassani, L. T. Taylor, and M. Agah, "MEMS-Based Multi-Inlet/Outlet Preconcentrator Coated by Inkjet Printing of Polymer Adsorbents," Sens. Actuators, B, 133 (1) pp. 24-32, 2008.

[8] E. H. M. Camara, P. Breuil, D. Briand, L. Guillot, C. Pijolat, and N. F. de Rooij, "A micro gas preconcentrator with improved performances for environmental monitoring," in Solid-State Sensors, Actuators and Microsystems Conference, 2009. TRANSDUCERS 2009. International, 2009, pp. 983 986.

[9] M. Agah, G. R. Lambertus, R. Sacks, and K. Wise, "Highspeed MEMS-based gas chromatography," Journal of Microelectromechanical Systems, 15 (5) pp. 1371-8, 2006.

[10] B. Alfeeli, M. Ashraf-Khorassani, L. T. Taylor, and M. Agah, "Multi-inlet/outlet Preconcentrator with 3-D m-structures Coated by Inkjet Printing of Tenax TA," in Solid-State Sensors, Actuators, and Microsystems Workshop, Hilton Head Island, South Carolina, 2008, pp. 118-121.

[11] M. Agah, J. A. Potkay, G. Lambertus, R. Sacks, and K. D. Wise, "High-Performance Temperature-Programmed Microfabricated Gas Chromatography Columns," Journal of Microelectromechanical Systems, 14 (5) pp. 1039-1050, 2005.

\section{CONTACT}

*B. Alfeeli, tel: +1-540-231-4180; alfeeli@vt.edu 